\title{
Clustering and Metaclustering with Nonnegative Matrix Decompositions
}

\author{
Liviu Badea \\ AI Lab, National Institute for Research and Development in Informatics, \\ 8-10 Averescu Blvd., Bucharest, Romania \\ badea@ici.ro
}

\begin{abstract}
Although very widely used in unsupervised data mining, most clustering methods are affected by the instability of the resulting clusters w.r.t. the initialization of the algorithm (as e.g. in k-means). Here we show that this problem can be elegantly and efficiently tackled by meta-clustering the clusters produced in several different runs of the algorithm, especially if "soft" clustering algorithms (such as Nonnegative Matrix Factorization) are used both at the object- and the meta-level. The essential difference w.r.t. other metaclustering approaches consists in the fact that our algorithm detects frequently occurring sub-clusters (rather than complete clusters) in the various runs, which allows it to outperform existing algorithms. Additionally, we show how to perform two-way meta-clustering, i.e. take both object and sample dimensions of clusters simultaneously into account, a feature which is essential e.g. for biclustering gene expression data, but has not been considered before.
\end{abstract}

\section{Introduction and Motivation}

Clustering is one of the most widely used unsupervised learning methods and the number of different clustering approaches is overwhelming. However, despite their wide variety, most clustering methods are affected by a common problem: the instability of the resulting clusters w.r.t. the initialization of the algorithm (as in the case of k-means) and w.r.t. slight differences in the input dataset as a result of resampling the initial data (e.g. in the case of hierarchical clustering). This is not surprising if we adopt a unifying view of clustering as a constrained optimization problem, since the fitness landscape of such a complex problem may involve many different local minima into which the algorithm may get caught when started off from different initial states.

Although such an instability seems hard to avoid, we may be interested in the clusters that keep reappearing in the majority of the runs of the algorithm. This is related to the problem of combining multiple clustering systems, which is the unsupervised analog of the classifier combination problem [8], a comparatively simpler problem that has attracted a lot of research in the past decade. Combining clustering results is more complicated than combining classifiers, as it involves solving an additional so-called cluster correspondence problem, which amounts to finding the best matches between clusters generated in different runs. 
The cluster correspondence problem can also be cast as an unsupervised optimization problem, which can be solved by a (meta-) clustering algorithm. Choosing an appropriate meta-clustering algorithm for dealing with this problem crucially depends on the precise notion of cluster correspondence.

A very strict notion of one-to-one correspondence between the clusters of each pair of clustering runs may be too tough to be realized in most practical cases. For example, due to the above-mentioned instability, different runs of k-means clustering with different initializations may easily produce different sets of clusters, e.g. $\operatorname{run}_{1}=\{\{1,2,3\},\{4,5\}\}, \operatorname{run}_{2}=\{\{1,2\},\{3,4,5\}\}, \ldots$

A more lenient notion of cluster correspondence would look for clusters that keep reappearing in all runs (while ignoring the rest), but only very few (if any) such clusters may exist for a large enough number of runs.

An even less restrictive notion could be envisioned by looking for clusters that are most similar (although not necessarily identical) across all runs. This is closest to performing something like single-linkage hierarchical clustering on the sets of clusters produced in the various clustering runs, with the additional constraint of allowing in each meta-cluster no more than a single cluster from each individual run. Unfortunately, this constraint will render the meta-clustering algorithm highly unstable. Thus, while trying to address the instability of (objectlevel) clustering using meta-level clustering, we end up with instability in the meta-clustering algorithm itself. Therefore, a "softer" notion of cluster correspondence is needed.

The main motivation for this work comes from genomics, more precisely from clustering gene expression data [2]. (Therefore, in the following we will frequently refer to clusters of genes rather than clusters of more abstract objects.) Most currently used clustering algorithms produce non-overlapping clusters, which represents a serious limitation in this domain, since a gene is typically involved in several biological processes. Here we adopt a biologically plausible simplifying assumption that the overlap of influences (biological processes) is additive

$$
X_{s g}=\sum_{c} X(s, g \mid c)
$$

where $X_{s g}$ is the expression level of gene $g$ in data sample $s$, while $X(s, g \mid c)$ is the expression level of $g$ in $s$ due to biological process $c$. We also assume that $X(s, g \mid c)$ is multiplicatively decomposable into the expression level $A_{s c}$ of the biological process (cluster) $c$ in sample $s$ and the membership degree $S_{c g}$ of gene $g$ in $c: \quad X(s, g \mid c)=A_{s c} \cdot S_{c g}$

\section{Nonnegative Matrix Factorization as a Soft Clustering Method}

Combining (1) and (2) leads to the reformulation of our clustering problem as a Nonnegative Matrix Factorization (of the $n_{s} \times n_{g}$ matrix $X$ as a product of an $n_{s} \times n_{c}$ matrix $A$ and an $n_{c} \times n_{g}$ matrix $\left.S\right)$ :

$$
X_{s g} \approx \sum_{c} A_{s c} \cdot S_{c g}
$$


with the additional nonnegativity constraints: $A_{s c} \geq 0, S_{c g} \geq 0$

(Expression levels and membership degrees cannot be negative.)

Such a problem can be cast as a constrained optimization problem:

minimize $C(A, S)=\frac{1}{2}\|X-A \cdot S\|_{F}^{2}=\frac{1}{2} \sum_{s, g}(X-A \cdot S)_{s g}^{2}$

subject to the nonnegativity constraints (4), and could be solved using Lee and Seung's Nonnegative Matrix Factorization (NMF) algorithm [4,5].

As explained above, such a factorization can be viewed as a "soft" clustering algorithm allowing for overlapping clusters, since we may have several significant $S_{c g}$ entries on a given column $g$ of $S$ (so a gene $g$ may "belong" to several clusters $c$ ).

Allowing for cluster overlap alleviates but does not completely eliminate the instability of clustering, since the optimization problem (5), (4) is non-convex.

In particular, the NMF algorithm produces different factorizations (biclusters) $\left(A^{(i)}, S^{(i)}\right)$ for different initializations and meta-clustering the resulting "soft" clusters $S^{(i)}$ could be used to obtain a more stable set of clusters.

However, using a "hard" meta-clustering algorithm would once again entail an unwanted instability. Therefore, we propose using Nonnegative Matrix Factorization as a "soft" meta-clustering approach.

This not only alleviates the instability of a "hard" meta-clustering algorithm, but also produces a "base" set of "cluster prototypes", out of which all clusters of all individual runs can be recomposed, despite the fact that they may not correspond to identically reoccurring clusters in all individual runs (see Figure 1).

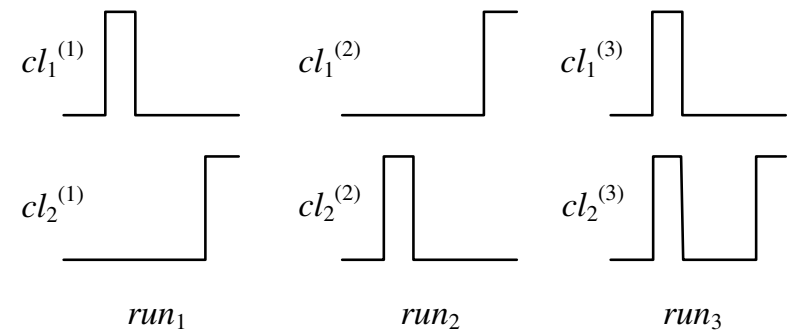

Fig. 1. Clusters obtained in different runs are typically combinations of a "base" set of "cluster prototypes" (rather than identical across all runs)

\section{Metaclustering with NMF}

We propose using NMF both for object-level clustering and meta-clustering. This unified approach solves in an elegant manner both the clustering and the cluster correspondence problem. More precisely, we first run NMF as object-level clustering $r$ times:

$$
X \approx A^{(i)} \cdot S^{(i)} \quad i=1, \ldots, r
$$

where $X$ is the data matrix to be factorized (samples $\times$ objects to be factorized), $A^{(i)}$ (samples $\times$ clusters) and $S^{(i)}$ (clusters $\times$ objects). 
To allow the comparison of membership degrees $S_{c g}$ for different clusters $c$, we scale the rows of $S^{(i)}$ to unit norm by taking advantage of the scaling invariance of the above factorization (6). More precisely:

Proposition. The NMF objective function (5) is invariant under the transformation $A \leftarrow A \cdot D, S \leftarrow D^{-1} \cdot S$, where $D=\operatorname{diag}\left(d_{l}, \ldots, d_{n c}\right)$ is a positive diagonal matrix.

Since a diagonal matrix $D$ operates on the rows of $S$ and on the columns of $A$, we can scale the rows of $S$ to unit norm by using a diagonal scaling with $d_{c}=\sqrt{\sum_{g} S_{c g}^{2}}$.

Next, we build a global $S$-matrix of size $r \cdot n_{c} \times n_{g}$ :

$$
S^{G}=\left(\begin{array}{c}
S^{(1)} \\
\vdots \\
S^{(r)}
\end{array}\right)
$$

by collecting all clusters (i.e. rows of $S^{(i)}$ ) from all runs and then use NMF once more to meta-cluster these clusters (i.e. the rows of $S^{G}$ ):

$$
S^{G} \approx \alpha \cdot \gamma
$$

where $\alpha$ and $\gamma$ are of sizes $r \cdot n_{c} \times n_{c}$ and $n_{c} \times n_{g}$ respectively. Note that whereas object level NMF clusters columns of $X$ (e.g. genes in our genomics application), meta-clustering clusters rows of $S^{G}$.

Note that $\alpha$ encodes the cluster-metacluster correspondence. On the other hand, the rows of $\gamma$ make up a base set of cluster prototypes, out of which all clusters of all individual runs can be recomposed:

$$
S_{c}^{(i)}=\sum_{m} \alpha_{c+(i-1) n_{c}, m} \cdot \gamma_{m}
$$

where $S_{c}^{(i)}$ is the row $c$ of $S^{(i)}$, while $\gamma_{m}$ is the row $m$ of $\gamma$.

Using the notation $\alpha_{c m}^{(i)}$ for $\alpha_{c+(i-1) n_{c}, m}$, we can rewrite (9) as

$$
S_{c}^{(i)}=\sum_{m} \alpha_{c m}^{(i)} \cdot \gamma_{m}
$$

Ideally (in case of a perfect one-to-one correspondence of clusters across runs), we would expect the rows of $\alpha$ to contain a single significant entry $\alpha_{c, m(i, c)}^{(i)}$, so that each cluster $S_{c}^{(i)}$ corresponds to a single cluster prototype $\gamma_{m(i, c)}$ (where $m(i, c)$ is a function of $i$ and $c): S_{c}^{(i)}=\alpha_{c, m(i, c)}^{(i)} \cdot \gamma_{m(i, c)}$

Additionally, each meta-cluster $m$ should contain no more than a single cluster from each individual run, i.e. there should be no significant entries $\alpha_{c^{\prime} m}^{(i)}$ and $\alpha_{c^{\prime \prime} m}^{(i)}$ with $c^{\prime} \neq c^{\prime \prime}$. Although it could be easily solved by a hard meta-clustering algorithm, such an ideal cluster correspondence is only very seldom encountered in practice, mainly due to the instability of most clustering algorithms. 
Thus, instead of such a perfect correspondence (10), we settle for a weaker one (9) in which the rows of $\alpha$ can contain several significant entries, so that all clusters (rows of $S^{G}$ ) are recovered as combinations of cluster prototypes (rows of $\gamma$ ).

The nonnegativity constraints of NMF meta-clustering are essential both for allowing the interpretation of $\gamma$ as cluster prototypes as well as for obtaining sparse factorizations $(\alpha, \gamma)$. (Experimentally, the rows of $\alpha$ tend to contain typically one or only very few significant entries.)

In order to make the prototype clusters (rows of $\gamma$ ) directly comparable to the clusters (rows) from $S^{G}$, we use the diagonal scaling

$$
\alpha \leftarrow \alpha \cdot D^{-1}, \quad \gamma \leftarrow D \cdot \gamma \quad \text { with } D=\operatorname{diag}\left(\frac{1}{r} \sum_{j} \alpha_{j m}\right) \text {. }
$$

The cluster prototypes matrix $\gamma$ produced by meta-clustering (8) is subsequently used as seed for a final NMF run aiming at producing the final factorization. More precisely, the seed for the final NMF run is $\left(A_{0}, \gamma\right)$, where $A_{0}$ is the nonnegative least squares solution to $X \approx A_{0} \cdot \gamma$.

We thus obtain a final factorization (3), which can be interpreted as a stable clustering of $X$ allowing for overlapping clusters. The algorithm is summarized below.

\section{Clustering with Metaclustering $(\mathbf{X}) \rightarrow(\mathbf{A}, \mathbf{S})$}

for $i=1, \ldots, r$

run $\operatorname{NMF}\left(X, A^{(0 i)}, S^{(0 i)}\right)$ with random initial matrices $A^{(0 i)}, S^{(0 i)}$ to produce a factorization with $n_{c}$ clusters: $X \approx A^{(i)} \cdot S^{(i)}$

scale the rows of $S^{(i)}$ to unit norm:

$$
A^{(i)} \leftarrow A^{(i)} \cdot D, S^{(i)} \leftarrow D^{-1} \cdot S^{(i)} \text { with } D=\operatorname{diag}\left(\sqrt{\sum_{g} S_{c g}^{2}}\right)
$$

end

Construct $S^{G}=\left(\begin{array}{c}S^{(1)} \\ \vdots \\ S^{(r)}\end{array}\right)$ and use $\operatorname{NMF}\left(S^{G}, \alpha^{(0)}, \gamma^{(0)}\right)$ (with random $\left.\alpha^{(0)}, \gamma^{(0)}\right)$ to

produce a factorization ("meta-clustering") with internal dimensionality $n_{c}$ : $S^{G} \approx \alpha \cdot \gamma$

scale the columns of $\alpha . \quad \alpha \leftarrow \alpha \cdot D^{-1}, \quad \gamma \leftarrow D \cdot \gamma \quad$ with $D=\operatorname{diag}\left(\sum_{j} \alpha_{j m} / r\right)$

Let $A_{0}$ be the nonnegative least squares solution to $X \approx A_{0} \cdot \gamma$

$\operatorname{Run} \operatorname{NMF}\left(X, A_{0}, \gamma\right)$ to produce the final factorization $X \approx A \cdot S$

$\underline{\operatorname{NMF}\left(X, A_{0}\right.} \underline{\underline{S_{0}}} \underline{\underline{0}} \underline{\rightarrow(A, S)}$

$A \leftarrow A_{0}, S \leftarrow S_{0}$

loop $\quad S_{c g} \leftarrow S_{c g} \frac{\left(A^{T} \cdot X\right)_{c g}}{\left(A^{T} \cdot A \cdot S\right)_{c g}}$ 


$$
A_{s c} \leftarrow A_{s c} \frac{\left(X \cdot S^{T}\right)_{s c}}{\left(A \cdot S \cdot S^{T}\right)_{s c}}
$$

until convergence.

\section{Sparser Decompositions}

Although NMF tends to produce sparse factorizations that are quite immune to moderate levels of noise [4], even sparser decompositions may be desired to cope with higher noise levels. An ad-hoc approach to obtaining such sparser factorizations would fix to zero all the elements below a given threshold of an NMF factorization and then apply several re-optimization rounds until a fixpoint is attained. Hoyer's Nonnegative Sparse Coding (NNSC) algorithm [3] is a more elegant approach that factorizes $X \approx A \cdot S$ by optimizing an objective function that combines the fit of the factorization to the original data with a size term penalizing the non-zero entries of $S$ :

$$
\text { minimize } \quad C(A, S)=\frac{1}{2}\|X-A S\|_{F}^{2}+\lambda \sum_{c, g} S_{c g}
$$

subject to the nonnegativity constraints $A_{s c} \geq 0, S_{c g} \geq 0$.

(NMF is recovered by setting the size parameter $\lambda$ to zero, while a non-zero $\lambda$ would lead to sparser factorizations.) Unfortunately however, the scaling invariance of the fitness term $\frac{1}{2}\|X-A S\|_{F}^{2}$ makes the size term ineffective, since the latter can be forced as small as needed by using a diagonal scaling $D$ with small enough entries. Additional constraints are therefore needed to render the size term operational. Since a diagonal matrix $D$ operates on the rows of $S$ and on the columns of $A$, we could impose unit norms either for the rows of $S$, or for the columns of $A$.

Unfortunately, the objective function (11) used in [3] produces decompositions that depend on the scale of the original matrix $X$ (i.e. the decompositions of $X$ and $\eta X$ are essentially different), regardless of the normalization scheme employed. For example, if we constrain the rows of $S$ to unit norm, then we cannot have decompositions of the form $X \approx A \cdot S$ and $\eta X \approx \eta A \cdot S$, since at least one of these is in general non-optimal due to the dimensional inhomogeneity of the objective function w.r.t. $A$ and $X: C_{\eta X}(\eta A, S)=\eta^{2} \frac{1}{2}\|X-A S\|_{F}^{2}+\lambda \sum_{c, g} S_{c g}$. On the other hand, if we constrain the columns of $A$ to unit norm, the decompositions $X \approx A \cdot S$ and $\eta X \approx A \cdot \eta S$ cannot be both optimal, again due to the dimensional inhomogeneity of $C, \quad$ now w.r.t. $S \quad$ and $X: C_{\eta X}(A, \eta S)=\eta^{2} \frac{1}{2}\|X-A S\|_{F}^{2}+\eta \lambda \sum_{c, g} S_{c g}$.

Therefore, as long as the size term depends only on $S$, we are forced to constrain the columns of $A$ to unit norm, while employing an objective function that is dimensionally homogeneous in $S$ and $X$. One such dimensionally homogeneous objective function is: 


$$
C(A, S)=\frac{1}{2}\|X-A S\|_{F}^{2}+\lambda\|S\|_{F}^{2}
$$

which will be minimized subject to the nonnegativity constraints and the constraints on the norm of the columns of $A$ : $\left\|A_{c}\right\|=1$ (i.e. $\sum_{s} A_{s c}^{2}=1$ ).

It can be easily verified that this produces scale independent decompositions, i.e. if $X \approx A \cdot S$ is an optimal decomposition of $X$, then $\eta X \approx A \cdot \eta S$ is an optimal decomposition of $\eta X$.

The constrained optimization problem could be solved with a gradient-based method. However, in the case of NMF, faster so-called "multiplicative update rules" exist [5,3], which we have modified for the NNSC problem as follows.

\section{Modified NNSC algorithm}

Start with random initial matrices $A$ and $S$

$$
\text { loop } \begin{gathered}
S_{c g} \leftarrow S_{c g} \frac{\left(A^{T} \cdot X\right)_{c g}}{\left(A^{T} \cdot A \cdot S+\lambda S\right)_{c g}} \\
A \leftarrow A+\mu(X-A \cdot S) \cdot S^{T}
\end{gathered}
$$

normalize the columns of $A$ to unit norm: $A \leftarrow A \cdot D^{-1}, D=\operatorname{diag}\left(\sqrt{\sum_{s} A_{s c}^{2}}\right)$

until convergence.

Note that we factorize $X$ rather than $X^{\mathrm{T}}$ since the sparsity constraint should affect the clusters of genes (i.e. $S$ ) rather than the clusters of samples $A$. (This is unlike NMF, for which the factorizations of $X$ and $X^{\mathrm{T}}$ are symmetrical.)

\subsection{Sparser Factorizations and Noise}

To demonstrate that sparser factorizations are better at coping with noise than simple NMF, we generated a synthetic dataset with highly overlapping clusters and very large additive noise (the standard deviation of the noise was 0.75 of the standard deviation of the original data).

We ran our modified NNSC algorithm with increasingly larger $\lambda$ (ranging from 0 to 0.75 ) and observed that the gene clusters were recovered almost perfectly despite the very large noise, especially for small values of $\lambda$.

Figure 2 shows the original data without and with noise respectively (upper row), as well as the reconstructed data $(A \cdot S)$ for $\lambda=0.05$ and $\lambda=0.75$ (lower row). Note that the reconstructed data is closer to the original noise-free data than to the noisy original. The following Table shows the relative error computed w.r.t. the noisy data $X_{\text {noisy }}$ (i.e. $\varepsilon_{\text {noisy }}=\left\|X_{\text {noisy }}-A S\right\|_{F} /\left\|X_{\text {nois }}\right\|_{F}$ ) as well as the relative error w.r.t. the original data $X_{\text {orig }}$ (before adding noise, i.e. $\varepsilon_{\text {orig }}=\left\|X_{\text {orig }}-A S\right\|_{F} /\left\|X_{\text {orig }}\right\|_{F}$ ) for several values of $\lambda$ ranging from 0 to 0.75 .

\begin{tabular}{|c|c|c|c|c|c|c|c|c|}
\hline$\lambda$ & 0 & 0.02 & 0.05 & 0.1 & 0.2 & 0.4 & 0.5 & 0.75 \\
\hline$\varepsilon_{\text {noisy }}$ & 0.2001 & 0.2017 & 0.2053 & 0.2161 & 0.2295 & 0.2452 & 0.2983 & 0.3228 \\
\hline$\varepsilon_{\text {orig }}$ & 0.1403 & 0.1375 & $\mathbf{0 . 1 3 6 4}$ & 0.1419 & 0.1544 & 0.1719 & 0.2323 & 0.2604 \\
\hline
\end{tabular}



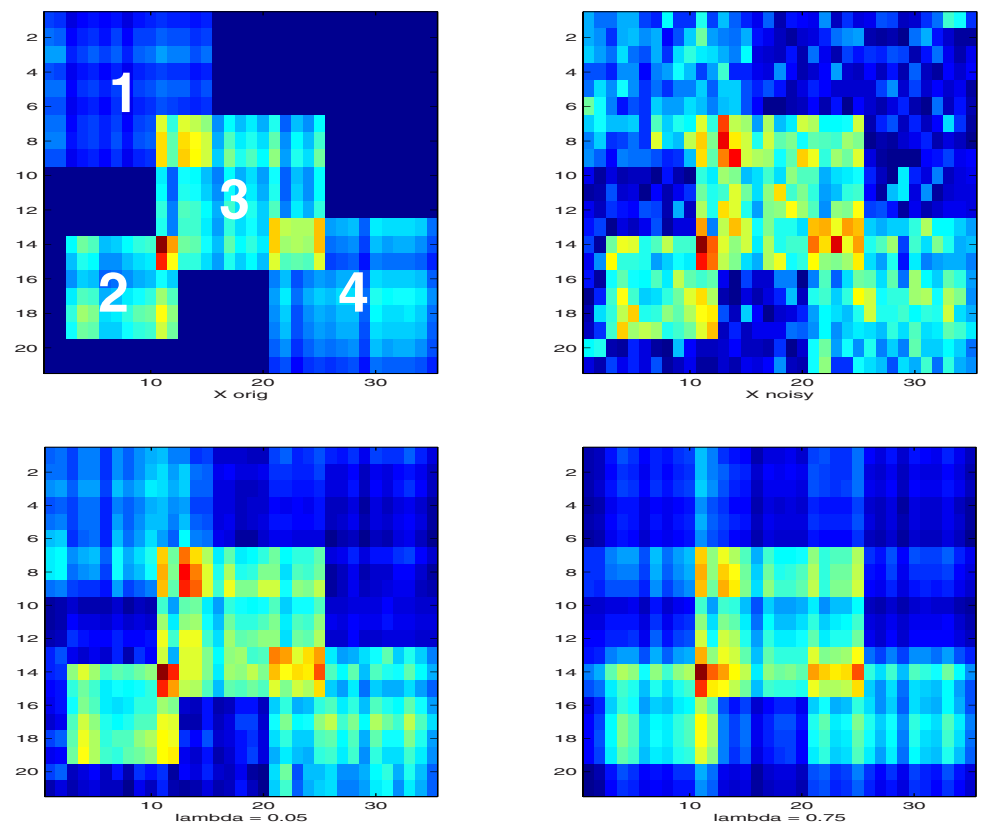

Fig. 2. A synthetic dataset

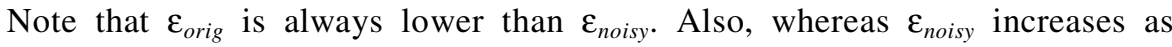
expected with $\lambda, \varepsilon_{\text {orig }}$ attains a minimum at $\lambda=0.05$ showing that small values of $\lambda$ tend to improve not only the clusters, but also the error w.r.t. the original data.

\section{Two-Way Meta-clustering}

The meta-clustering approach based on (8) takes only the gene clusters (rows of $S^{(i)}$ ) into account. Although this works very well in many cases, it will fail whenever two clusters correspond to very similar sets of genes, while differing along the sample dimension. For example, clusters 1 and 2 from Figure 2 are quite similar along the gene dimension, so a meta-clustering method looking just at genes would be incapable of discriminating between the two clusters, unless it also looks at the "sample clusters" $A^{(i)}$. In the following, we show that a slight generalization of NMF, namely Positive Tensor Factorization (PTF) [6] can be successfully used to perform two-way meta-clustering, which takes both the gene and the sample dimensions into account. (As far as we know, this elegant view of metaclustering as a PTF problem has not been considered before.)

Naively, one would be tempted to try clustering the biclusters ${ }^{1} A_{c}^{(i)} \cdot S_{c}^{(i)}$ instead of the gene clusters $S_{c}^{(i)}$, but this is practically infeasible in most real-life datasets because it involves factorizing a matrix of size $r \cdot n_{c} \times n_{s} \cdot n_{g}$. On closer inspection,

${ }^{1} A_{c}^{(i)}$ is the column $c$ of $A^{(i)}$, while $S_{c}^{(i)}$ is the row $c$ of $S^{(i)}$. 
however, it turns out that it is not necessary to construct this full-blown matrix actually we are searching for a Positive Tensor Factorization of this matrix ${ }^{2}$

$$
A_{s c}^{(i)} \cdot S_{c g}^{(i)} \approx \sum_{k=1}^{n_{c}} \alpha_{c k}^{(i)} \cdot \beta_{s k} \cdot \gamma_{k g}
$$

The indices in (14) have the following domains: $s$ - samples, $g$ - genes, $c-$ clusters, $k$ - metaclusters. To simplify the notation, we could merge the indices $i$ and $c$ into a single index $(i c)$, so that the factorization becomes:

$$
A_{s(i c)} \cdot S_{(i c) g} \approx \sum_{k=1}^{n_{c}} \alpha_{(i c) k} \cdot \beta_{s k} \cdot \gamma_{k g}
$$

Note that $\beta$ and $\gamma$ are the "unified" versions of $A^{(i)}$ and $S^{(i)}$ respectively, while $\alpha$ encodes the cluster-metacluster correspondence.

The factorization (14') can be computed using the following multiplicative update rules (the proofs are straightforward generalizations of those for NMF and can also be found e.g. in [6]):

$$
\begin{aligned}
& \alpha \leftarrow \alpha * \frac{\left(A^{T} \cdot \beta\right) *\left(S \cdot \gamma^{T}\right)}{\alpha \cdot\left[\left(\beta^{T} \cdot \beta\right) *\left(\gamma \cdot \gamma^{T}\right)\right]} \\
& \beta \leftarrow \beta * \frac{A \cdot\left[\alpha *\left(S \cdot \gamma^{T}\right)\right]}{\beta \cdot\left[\left(\alpha^{T} \cdot \alpha\right) *\left(\gamma \cdot \gamma^{T}\right)\right]} \\
& \gamma \leftarrow \gamma * \frac{\left[\alpha *\left(A^{T} \cdot \beta\right)\right]^{T} \cdot S}{\left[\left(\alpha^{T} \cdot \alpha\right) *\left(\beta^{T} \cdot \beta\right)\right]^{T} \cdot \gamma}
\end{aligned}
$$

where ' $*$ ' and '--' denote element-wise multiplication and division of matrices, while '.' is ordinary matrix multiplication.

The PTF factorization (14') should be contrasted with our previous metaclustering approach (8) based on NMF:

$$
S_{c g}^{(i)} \approx \sum_{k=1}^{n_{c}} \alpha_{c k}^{(i)} \cdot \gamma_{k g}
$$

It can be easily seen that whereas (14) groups biclusters by taking both the gene and the sample dimension into account, (8') may confuse two biclusters that have similar gene components (even if they have different sample supports). For example, (8') confuses biclusters 1 and 2 from Figure 2, while (14) is able to perfectly discriminate between the two despite the noise and the difference in intensity between the two biclusters.

After convergence of the PTF update rule, we normalize the rows of $\gamma$ to unit norm $\left(\left\|\gamma_{k}\right\|=1\right)$, as well as the columns of $\alpha$ such that $\sum_{i, c} \alpha_{c k}^{(i)}=r$ ( $r$ being the number of runs): ${ }^{3}$

${ }^{2}$ More precisely, we are dealing with the constrained optimization problem $\min C(\alpha, \beta, \gamma)=\frac{1}{2} \sum_{i, c, s, g}\left(A_{s c}^{(i)} S_{c g}^{(i)}-\sum_{k=1}^{n_{c}} \alpha_{c k}^{(i)} \beta_{s k} \gamma_{k g}\right)^{2}$ subject to $\alpha, \beta, \gamma \geq 0$.

${ }^{3}$ In order to be able to interpret $\beta$ and $\gamma$ as "unified" $A^{(i)}$ and $S^{(i)}$ respectively, we need to have $\sum_{c} \alpha_{c k}^{(i)} \approx 1$, i.e. $\sum_{i, c} \alpha_{c k}^{(i)} \approx r$, since $X \approx \sum_{c} A_{s c}^{(i)} \cdot S_{c g}^{(i)} \approx \sum_{k=1}^{n_{c}}\left(\sum_{c} \alpha_{c k}^{(i)}\right) \cdot \beta_{s k} \cdot \gamma_{k g}$. 


$$
\begin{gathered}
\gamma_{k g} \mapsto \frac{1}{\left\|\gamma_{k}\right\|} \cdot \gamma_{k g} \\
\alpha_{(i c) k} \mapsto \frac{r}{\sum_{i^{\prime}, c^{\prime}} \alpha_{\left(i^{\prime} c^{\prime}\right) k}} \cdot \alpha_{(i c) k} \\
\beta_{s k} \mapsto \frac{\left\|\gamma_{k}\right\|}{r} \cdot \sum_{i, c} \alpha_{(i c) k} \cdot \beta_{s k}
\end{gathered}
$$

and then run NMF initialized with $(\beta, \gamma)$ to produce the final factorization $X \approx A \cdot S$.

\section{Experimental Evaluation}

We evaluated our algorithm on synthetic datasets ${ }^{4}$ generated by continuous latent variable graphical models as in Figure 3. (The clusters corresponding to the latent variables $L_{k}$ overlap in the variables $X_{i}$ influenced by several $L_{k}$.) To generate nonnegative biclusters as in Figure 2, we set $L_{k}$ to nonzero values (drawn from the absolute of a normal distribution) only in certain subsets of samples. The observable variables $X_{i}$ were affected by additive normal noise with a standard deviation $\sigma\left(\varepsilon_{i}\right)=v \cdot \sigma\left(X_{i}^{(0)}\right)$ equal to a fraction $v$ of the standard deviation of the noise-free "signal" $X_{i}^{(0)}$. (It can be easily seen that this model is equivalent to $X=$ $A^{(0)} \cdot S^{(0)}+\varepsilon$, where $X=\left(X_{1} \ldots X_{n g}\right)$ and $S^{(0)}{ }_{k j}$ are the coefficients associated to the $L_{k} \rightarrow X_{j}$ edges.)

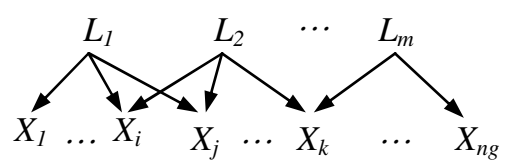

Fig. 3. Latent variable model for overlapping clusters

The Table below presents a comparison of various combinations of clustering and meta-clustering algorithms - columns of the Table correspond to clustering algorithms (k-means, fuzzy k-means [7] and NMF), while the rows are associated to meta-clustering algorithms (k-means, fuzzy k-means, NMF and PTF) with or without a final NMF step, as well as to the best individual clustering run (resampling). The figures in the Table represent average matches of the reconstructed clusters with the original ones, together with the associated relative errors (we display both averages and standard deviations for 10 runs of each metaclustering method with different input data $X$ ).

${ }^{4}$ The small dataset from Figure 2 with 21 samples and 35 genes allows a human analysis of the results. 
Defining the match between two sets of possibly overlapping clusters is nontrivial. For each cluster $C_{1}$ from clustering 1 , we determine the single cluster $C_{2}$ from clustering 2 into which it is best included, i.e. the one with the largest $\left|C_{1} \cap C_{2}\right| /\left|C_{1}\right|$. We proceed analogously for the clusters $C_{2}$ from clustering 2 . Then, for each cluster $C_{1}$ (from clustering 1), we determine its match $\left|C_{1} \cap C_{2}\right| /\left|C_{1} \cup C_{2}\right|$ with the union $C_{2}$ of clusters from clustering 2 , for which $C_{1}$ is the best including cluster (as determined in the previous step). Similarly, we determine matches for clusters $C_{2}$ from clustering 2. The average match of the two clusterings is then the mean of all these matches (for all $C_{1}$ and all $C_{2}$ ).

The Table clearly demonstrates the necessity of using nonnegative decompositions like NMF (either as individual runs or as the final step) for obtaining reasonable results. Indeed, the best match without any nonnegative decompositions is $55 \%$ and the lowest relative error 0.2 , whereas with nonnegative decompositions, we obtain a nearly perfect match $(98 \%)$ with a relative error of $10^{-3}$.

\begin{tabular}{|l|l|l|l|}
\hline $\begin{array}{l}\text { match (std match) } \\
\text { relative error (std error) }\end{array}$ & Kmeans & fcm & NMF \\
\hline kmeans(meta) & $\mathbf{0 . 5 3 ( 0 . 0 2 1 )}$ & $\mathbf{0 . 5 5 ( 0 . 0 5 8 )}$ & $\mathbf{0 . 8 1 ( 0 . 1 2 3 )}$ \\
& $0.306(0.032)$ & $0.2(0.018)$ & $0.052(0.033)$ \\
\hline kmeans(meta) + NMF(final) & $\mathbf{0 . 6 2 ( 0 . 0 5 6 )}$ & $\mathbf{0 . 6 3 ( 0 . 1 8 1 )}$ & $\mathbf{0 . 9 ( 0 . 1 4 8 )}$ \\
& $0.153(0.059)$ & $0.094(0.046)$ & $0.002(0.001)$ \\
\hline fcm(meta) & $\mathbf{0 . 5 1 ( 0 . 0 4 1 )}$ & $\mathbf{0 . 5 3 ( 0 . 0 1 1 )}$ & $\mathbf{0 . 9 2 ( 0 . 1 2 6 )}$ \\
\hline fcm(meta) + NMF(final) & $0.315(0.054)$ & $0.202(0.019)$ & $0.014(0.004)$ \\
\hline NMF(meta) & $0.05(\mathbf{0 . 1 7 8})$ & $\mathbf{0 . 5 6 ( 0 . 0 2 4 )}$ & $\mathbf{0 . 9 2 ( 0 . 1 2 6 )}$ \\
\hline NMF(meta) + NMF(final) & $\mathbf{0 . 5 ( 0 . 0 3 2 )}$ & $0.112(0.008)$ & $0.002(0)$ \\
\hline PTF(meta) & $0.313(0.042)$ & $\mathbf{0 . 5 3 ( 0 . 0 0 9 )}$ & $\mathbf{0 . 6 9 ( 0 . 0 0 8 )}$ \\
\hline PTF(meta) + NMF(final) & $0.132(0.016)$ & $\mathbf{0 . 1 9 4 ( 0 . 0 1 8 )}$ & $0.027(0.043)$ \\
\hline Best clustering run & $\mathbf{0 . 4 9 ( 0 . 0 4 4 )}$ & $0.119(0.012)$ & $\mathbf{0 . 7 4 ( 0 . 1 1 1 )}$ \\
(out of 10) & $0.1287(0.023)$ & $\mathbf{0 . 5 3 ( 0 . 0 1 )}$ & $0.012(0.025)$ \\
\hline & $\mathbf{0 . 4 9 ( 0 . 0 1 7 )}$ & $\mathbf{0 . 9 8}(0.037)$ \\
& $0.307(0.011)$ & $0.116(0.011)$ & $0.023(0.006)$ \\
\hline
\end{tabular}

Note that clustering runs based on NMF are far superior to other methods. On the other hand, all tested meta-clustering algorithms perform reasonably well (with PTF faring best), especially in terms of relative error. However, as already discussed in Section 5, meta-clustering with NMF does not recover the clusters very well (average matches are around $74 \%$ versus virtually perfect matches for PTF (98\%), 92\% for fuzzy k-means and about $90 \%$ for k-means+NMF). NMF and PTF on NMF runs are also quite stable (the std of the match is $0.8 \%$ and $4 \%$ respectively).

Also note that although meta-clustering does not always outperform the best individual run in terms of relative error, it does outperform it in terms of the match with the original clusters (98\% versus $76 \%)$.

We also considered larger problems in which the overlapping clusters can be discriminated by looking at the gene dimension only. As expected, in such cases the best results are obtained by a combination which uses NMF for metaclustering: (NMF, NMF, NMF). 
We also observed that k-means and fuzzy k-means are far inferior to NMF (as meta-clustering algorithms) in problems with a larger number of clusters. This is because, as the number of clusters increases, the fraction of perfectly reconstructed clusters in a limited number of runs decreases sharply. This makes meta-clustering algorithms like k-means or fuzzy k-means less effective, since these algorithms search for clusters that reoccur in a large fraction of runs. On the other hand, our approach using nonnegative decompositions looks for cluster prototypes out of which the clusters of all individual runs can be recomposed (recall Fig. 1) and therefore may behave well even with a limited number of runs (such as 10-20 in our experiments).

\section{Related Work and Conclusions}

Bradley and Fayyad [1] use $k$-means for meta-clustering a number of $k$-means runs on subsamples of the data for initializing a final k-means run. However, the use of a "hard" clustering approach like k-means in domains featuring overlapping biclusters produces dramatically less accurate results than our approach using NMF or PTF for meta-clustering NMF runs (53\% match and $30.6 \%$ error vs. $98 \%$ match and $0.1 \%$ error for our algorithm). ${ }^{5}$

The main technical contribution of this paper consists in showing how NMF and PTF can be used to solve the cluster correspondence problem for "soft" biclustering algorithms such as NMF (which is significantly more involved than the cluster correspondence problem for "hard" algorithms and, as far as we know, has not been addressed before). The present approach is significantly different from other biclustering approaches - for example Cheng's biclustering [9] is based on a simpler additive model that is not scale invariant (problematic in the case of gene expression data). Our algorithm not only significantly outperforms all existing approaches (especially in terms of recovering the original clusters), but - more importantly provides a conceptually elegant solution to the cluster correspondence problem. Furthermore, an initial application of the method to a large lung cancer dataset [10] proved computationally tractable and was able to perfectly recover the known histological classification of the various lung cancer types in the dataset. (For lack of space, we refer to the supplementary information at http://www.ai.ici.ro/ecml05/ meyerson.pdf). The genomics applications will be the subject of future research.

Acknowledgements. I am grateful to Doina Tilivea who helped in the experimental evaluation of the algorithms.

\section{References}

1. Bradley P.S., Fayyad U.M. Refining Initial Points for K-Means Clustering, Proc. ICML-98, pp. 91-99.

2. Eisen M.B., P.T. Spellman, P.O. Brown, D. Botstein. Cluster analysis and display of genome-wide expression patterns, PNAS Vol.95, 14863-8, Dec. 1998.

${ }^{5}$ However, it is fair to say that [1] did not aim at improving the stability of clustering as we do, but at handling large datasets. 
3. Hoyer P.O. Non-negative sparse coding. Neural Networks for Signal Processing XII, 557-565, Martigny, 2002.

4. Lee D.D., H.S. Seung. Learning the parts of objects by non-negative matrix factorization. Nature, vol. 401, no. 6755, pp. 788-791, 1999.

5. Lee D.D., H.S. Seung. Algorithms for non-negative matrix factorization. Proc. NIPS*2000, MIT Press, 2001.

6. Welling M., Weber M. Positive tensor factorization. Pattern Recognition Letters 22(12): 1255-1261 (2001).

7. Bezdek J.C. Pattern Recognition with Fuzzy Objective Function Algorithms. Plenum Press, 1981.

8. Bauer E. Kohavi R. An empirical comparison of voting classification algorithms: bagging, boosting, and variants. Machine Learning 36 (1999) 105-139.

9. Cheng Y. Church G. Biclustering of expression data. Proc. ISMB-2000, 93-103.

10. Bhattacharjee et al. Classification of human lung carcinomas by mRNA expression profiling reveals distinct adenocarcinoma subclasses. Proc. Natl. Acad. Sci. USA. 2001 Nov. 20;98(24):13790-5. 